\title{
Domain Knowledge-Assisted Automatic Diagnosis of Idiopathic Pulmonary Fibrosis (IPF) Using High Resolution Computed Tomography (HRCT) (Student Abstract)
}

\author{
Wenxi Yu, ${ }^{1,2}$ Hua Zhou, ${ }^{2}$ Jonathan G. Goldin, ${ }^{1}$ Grace Hyun J. Kim ${ }^{1,2}$ \\ ${ }^{1}$ Department of Radiological Sciences, David Geffen School of Medicine, University of California, Los Angeles \\ ${ }^{2}$ Department of Biostatistics, Fielding School of Public Health, University of California, Los Angeles \\ $\{$ w.yu, huazhou\}@ucla.edu, \{JGoldin, GraceKim\}@mednet.ucla.edu
}

\begin{abstract}
Domain knowledge acquired from pilot studies is important for medical diagnosis. This paper leverages the populationlevel domain knowledge based on the D-optimal design criterion to judiciously select CT slices that are meaningful for the disease diagnosis task. As an illustrative example, the diagnosis of idiopathic pulmonary fibrosis (IPF) among interstitial lung disease (ILD) patients is used for this work. IPF diagnosis is complicated and is subject to inter-observer variability. We aim to construct a time/memory-efficient IPF diagnosis model using high resolution computed tomography (HRCT) with domain knowledge-assisted data dimension reduction methods. Four two-dimensional convolutional neural network (2D-CNN) architectures (MobileNet, VGG16, ResNet, and DenseNet) are implemented for an automatic diagnosis of IPF among ILD patients. Axial lung CT images are acquired from five multi-center clinical trials, which sum up to 330 IPF patients and 650 non-IPF ILD patients. Model performance is evaluated using five-fold cross-validation. Depending on the model setup, MobileNet achieved satisfactory results with overall sensitivity, specificity, and accuracy greater than $90 \%$. Further evaluation of independent datasets is underway. Based on our knowledge, this is the first work that (1) uses population-level domain knowledge with optimal design criterion in selecting CT slices and (2) focuses on patient-level IPF diagnosis.
\end{abstract}

\section{Introduction}

Idiopathic pulmonary fibrosis (IPF) is defined as a specific form of chronic, progressive fibrosing interstitial pneumonia of unknown causes. It is a rare disease with irreversible and unpredictable nature, resulting in distinct patterns of survival (usually 2-5 years). The radiological diagnosis of IPF is challenging and is subject to inter-observer variability, even for experienced radiologists.

We aim to develop an efficient (memory-efficient and no lung segmentation is needed) and domain knowledgeassisted diagnostic model for IPF among ILD patients based on their axial lung CT scans. Prior knowledge and optimal design theory are incorporated in the data dimension reduction procedure to leverage the population-level IPF disease

Copyright (c) 2020, Association for the Advancement of Artificial Intelligence (www.aaai.org). All rights reserved. trends across the lung positions.

\section{Methods}

\section{Overview}

Our research problem is a binary classification task (IPF versus non-IPF), where the input are axial lung CT images of one patient visit. The output is a binary label $y \in\{0,1\}$ indicating whether the patient is IPF. HRCT imaging is acquired from five multi-center clinical trials with different CT scanners and protocols. In total, 330 IPF patients and 650 non-IPF ILD patients are used for this study.

An overview of this work is provided in Figure 1. Firstly, population-level domain knowledge is acquired from pilot studies. Secondly, based on the domain knowledge, we sample $24 \mathrm{CT}$ slice positions using weights proportional to the population-level median of IPF progression. Thirdly, we propose three methods to reduce data dimension from 24 slices to 3 slices to enable the use of 2D deep learning architectures: directly select the $5^{\text {th }}, 10^{\text {th }}$, and $15^{\text {th }}$ out of the 24 slices (proposal 1), bilinear interpolation (proposal 2), and D-optimal criterion guided slice selection (proposal 3). After data dimension reduction, transfer learning (TL) is incorporated by initializing model parameters with pre-trained weights on ImageNet. Further, multiple deep learning architectures are tested by five-fold cross-validation. Lastly, model diagnostics are performed by occlusion maps and class activation mapping (CAM).

\section{Domain Knowledge-Assisted Slice Sampling}

Prior studies used particle swarm optimization incorporated with a random forest method to predict the slice-level IPF progression status (progressive versus not progressive) (Shi et al. 2019). Based on the predictive results of the pilot study, we calculated the percentage of progressive lung area pixels across the CT slice position.

D-optimal Criterion Suppose the statistical model of interest can be written in the form of $y_{i}=f\left(x_{i}\right)^{T} \beta+\epsilon_{i}, i=$ $1, \ldots, N$. Here $y_{i}$ is the variable of interest, $f\left(x_{i}\right)$ is a design vector of size $p \times 1$ and $N$ is the sample size. $\beta$ is the unknown parameter of size $p \times 1$ and the error term follows normal distribution $\epsilon_{i} \sim N\left(0, \sigma^{2}\right)$. D-optimal criterion is 


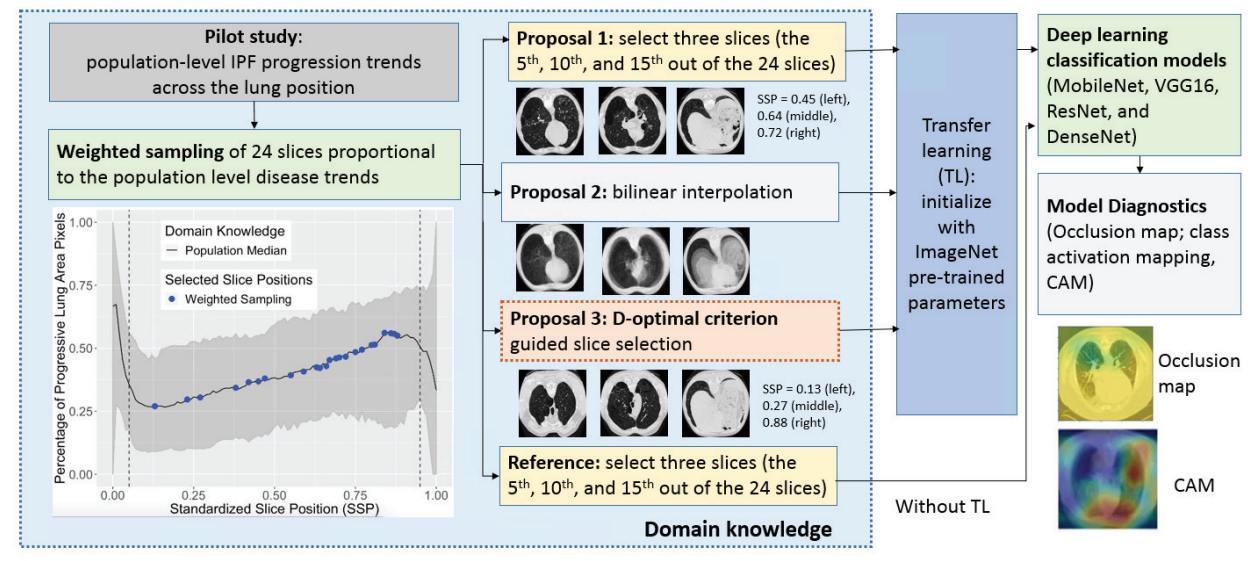

Figure 1: Flowchart of the Study Design

minimizing $\operatorname{Det}[\operatorname{Cov}(\beta)]$. Thus, collecting points based on D-optimal criterion can estimate model parameters with the smallest volume of the confidence ellipsoid.

We model the population-level percentage of lung area progressive pixels versus standardized slice position (SSP, the formula provided in the supplementary materials) under generalized linear model with logit link functions for this task. Cubic, quartic and fractional polynomial (FP) models are applied for this task to model the population trends. More details regarding the model selection and D-optimal solutions can be found in the supplementary materials.

\section{Deep Learning Model Architecture}

Four well-developed 2D-CNN structures, namely MobileNet (Howard et al. 2017), VGG16 (Simonyan and Zisserman 2014), ResNet (He et al. 2016) and DenseNet (Huang et al. 2017), are used for this IPF diagnosis task. Results are evaluated by five-fold cross-validation. More details regarding image preprocessing steps, model construction (hyperparameters, architectures and cross-validation) are provided in the supplementary materials.

\section{Model Diagnostics}

Occlusion maps and class activation mapping are used to identify the significant areas in the IPF diagnosis task.

\section{Results and Conclusions}

Table 1 summarizes the accuracy of each study based on different proposals under MobileNet. Model performance under other deep learning architectures (VGG16, ResNet, and DenseNet) is provided in supplementary materials.

We developed an efficient model using both transfer learning and domain knowledge by optimizing the selection of images using population-level information. This study shows the satisfactory model performance in the example of IPF diagnosis using CT images.

\section{Acknowledgments}

This research is supported by NIH, NHLBI-R21-HL140465.
Table 1: Five study-wise model performance by proposals using MobileNet with mean and standard deviations (SD) across five simulations. Note that Study 1 and 2 include IPF patients, reporting sensitivity. Similarly, Study 3, 4, and 5 contain non-IPF ILD patients, reporting specificity.

\begin{tabular}{cccccc}
\hline & \multicolumn{2}{c}{ Sensitivity } & \multicolumn{3}{c}{ Specificity } \\
& (IPF patients) & \multicolumn{2}{c}{ (Non-IPF ILD patients) } \\
\hline \multirow{3}{*}{ Proposal } & 1 & 2 & 3 & 4 & 5 \\
& mean & mean & mean & mean & mean \\
& (SD) & (SD) & (SD) & (SD) & (SD) \\
\hline \multirow{2}{*}{1} & 0.97 & 0.92 & 0.95 & 0.98 & 0.98 \\
& $(0.03)$ & $(0.04)$ & $(0.06)$ & $(0.02)$ & $(0.03)$ \\
2 & 0.96 & 0.89 & 0.96 & 0.98 & 0.99 \\
& $(0.10)$ & $(0.08)$ & $(0.04)$ & $(0.01)$ & $(0.03)$ \\
3 & 0.97 & 0.91 & 0.97 & 0.98 & 0.99 \\
Reference & $(0.04)$ & $(0.03)$ & $(0.01)$ & $(0.02)$ & $(0.01)$ \\
& 0.62 & 0.53 & 0.87 & 0.91 & 0.94 \\
& $(0.21)$ & $(0.15)$ & $(0.11)$ & $(0.09)$ & $(0.09)$ \\
\hline
\end{tabular}

\section{References}

He, K.; Zhang, X.; Ren, S.; and Sun, J. 2016. Deep residual learning for image recognition. In Proceedings of the IEEE conference on computer vision and pattern recognition, 770-778.

Howard, A. G.; Zhu, M.; Chen, B.; Kalenichenko, D.; Wang, W.; Weyand, T.; Andreetto, M.; and Adam, H. 2017. Mobilenets: Efficient convolutional neural networks for mobile vision applications. arXiv preprint arXiv:1704.04861.

Huang, G.; Liu, Z.; Van Der Maaten, L.; and Weinberger, K. Q. 2017. Densely connected convolutional networks. In Proceedings of the IEEE conference on computer vision and pattern recognition, 4700-4708.

Shi, Y.; Wong, W. K.; Goldin, J. G.; Brown, M. S.; and Kim, G. H. J. 2019. Prediction of progression in idiopathic pulmonary fibrosis using ct scans at baseline: A quantum particle swarm optimization-random forest approach. Artificial Intelligence in Medicine 101709.

Simonyan, K., and Zisserman, A. 2014. Very deep convolutional networks for large-scale image recognition. arXiv preprint arXiv:1409.1556. 